\title{
Whether coordinative (soccer) exercise improves executive functioning in kindergarten children has yet to be demonstrated
}

\author{
Adele Diamond
}

Received: 26 February 2014 / Accepted: 17 March 2014 / Published online: 12 April 2014

(C) Springer-Verlag Berlin Heidelberg 2014

I was excited to read this paper because I believe that exercise that requires thought, careful attention, and fast, flexible responding to the unexpected (as soccer practice should) should help improve executive functions. However, despite the claims of the title and abstract, sadly nothing about the benefits of soccer exercise for executive functions can be concluded from this paper.

A researcher needs a differential effect (a group $\times$ change interaction) to conclude that an activity or intervention mattered. Suppose a researcher gave all study participants a passage to read at Time 1 and then gave them the same passage to read at Time 2, and they read it faster at Time 2. If the researcher had them wash dishes between Times 1 and 2 , would he be justified in concluding that washing dishes improves reading speed? Of course not. The same problem unfortunately applies to the Chang et al. (2013) study. They found no differential effect. Therefore, we have no idea whether improvements were due to pre- and post-test practice effects or to the soccer exercises the children did in the interim between testings.

If children in one exercise group had improved more than children in the other exercise group, we could conclude that intensity of exercise mattered. However, children in both groups (high- and low-intensity soccer exercise) improved on the executive function task, with almost identical post-test results and pre-to-post improvement in both groups. Had there been a no-exercise group, and that group improved less than those who exercised, one could conclude that exercise mattered. Alas, there was no no-exercise control group. Given that children in the only two groups studied improved comparably, we have no idea if what they did between testing times 1 and 2 caused the improvement, or the improvement was simply due to practice effects from having taken the tests before.

Finding that participants do better after an intervention is insufficient to determine whether the intervention helped. We have no way of knowing whether comparable improvements might have been seen without the intervention. Thus, Chang et al. (2013) were incorrect to conclude that the "exercise intervention, regardless of intensity, resulted in shorter reaction times and higher response accuracy." To conclude that, they would have needed a third group that did something else and did not show comparable improvements on their outcome measure. I sincerely wish that this paper provided evidence of the benefits of soccer exercise for children's cognitive development, but alas it does not.

\footnotetext{
A. Diamond $(\bowtie)$

Canada Research Chair Tier 1, Department of Psychiatry, University of British Columbia (UBC), Vancouver, BC, Canada

e-mail: adele.diamond@ubc.ca
} 